\title{
Marker-assisted selection of new barley genotypes accumulating anthocyanins in grain
}

\author{
Kukoeva T.V. ${ }^{*}$, Generalova G.V. ${ }^{1}$, Strygina K.V. ${ }^{2}$, Grigoriev Yu.N. ${ }^{1}$, \\ Glagoleva A.Yu. ${ }^{1}$, Yakovlev M.A. ${ }^{2}$, Khlestkina E.K. ${ }^{1,3}$, Shoeva O.Yu. ${ }^{1}$ \\ ${ }^{1}$ Institute of Cytology and Genetics, SB RAS, Novosibirsk, Russia \\ ${ }^{2}$ Novosibirsk State Agrarian University, Novosibirsk, Russia \\ ${ }^{3}$ N.I. Vavilov All-Russian Institute of Plant Genetic Resources (VIR), St. Petersburg, Russia \\ *e-mail:kukoeva@bionet.nsc.ru
}

Barley (Hordeum vulgare L.) is an agronomically important crop. In the structure of world grain production, it ranks fourth after corn, wheat and rice. Barley grain contains a lot of fiber and little starch, which makes this culture a promising dietary food. To increase the nutritional value of grain crops, there is a steady world trend of saturation of grain with biologically active compounds anthocyanins. In barley, these compounds can be accumulated in pericarp (where their biosynthesis is controlled by the complementarily genes $A n t 1$ and $A n t 2)$, in the aleurone layer (HvMyc2), or in two tissues simultaneously, giving the grain a purple, blue and dark purple color, respectively. However, to date, barley varieties with colored grain have not been created and are not cultivated in our country. The purpose of this work was a marker-assisted breeding of new barley genotypes, accumulating anthocyanins in aleurone layer and pericarp based on cultivated Siberian varieties. The varieties Vorsinsky 2, Aley and Tanay were chosen as maternal forms. The near-isogenic lines of cultivar Bowman "Intence blue aleurone" and "Purple lemma and pericarp" (obtained from the Nordic Gene Bank), which are donors of the HvMyc2 and Ant1/Ant 2 genes, respectively, were chosen as paternal forms. The $\mathrm{F}_{1}$ hybrids were self-pollinated to get $\mathrm{F}_{2}$ plants. Among $\mathrm{F}_{2}$ offsprings, homozygous plants with blue and purple grain color were selected using diagnostic PCR markers (AFLP, CAPS and STS) for the $H v M y c 2$ and Ant1/Ant2 genes. These plants were sown in the field, where they were backcrossed with the original Siberian varieties. During the year, $350 \mathrm{BC}_{1} \mathrm{~F}_{2}$ plants (backcross of the first generation) were obtained, which will be subjected to a further 5-6 fold backcrossing and a marker-selection selection of homozygous plants.

Acknowledgements: The study was partially supported by the RFBR No. 19-016-00140 and ICG project No. 0324-2019-0039. 